\title{
Deciphering the driving forces in crystal packings by analysis of hydrogen bonds, electrostatic energies and contact enrichment ratios.
}

\author{
C. Jelsch ${ }^{1}$, B. Guillot ${ }^{1}$, Y. Bibila Mayaya Bisseyou ${ }^{2}$ \\ ${ }^{1}$ CRM2, CNRS, Lorraine University, Vandoeuvre les Nancy, France \\ ${ }^{2}$ University Felix Houphouet-Boigny, Abidjan, Côte d'Ivoire
}

christian.jelsch@univ-lorraine.fr

The decomposition of the crystal contacts on the Hirshfeld surface between pairs of interacting chemical species enables to derive a contact enrichment ratio $[1,2,3]$. This descriptor yields information on the propensity of chemical species to interact with themselves and other species. The enrichment ratio is obtained by comparing the actual and equiprobable contacts. $\mathrm{H} \cdots \mathrm{N}, \mathrm{H} \cdots \mathrm{O}$ and $\mathrm{H} \cdots \mathrm{S}$ as well as weak $\mathrm{H} \cdots$ halogen hydrogen bonds appear generally more or less enriched, depending on the context. Larges series of molecules made of a set of chemical groups and retrieved from the Cambridge Structural Database can be investigated to find trends in the propensity of interactions to form.

The electrostatic energy of between atoms in contact was also computed using a multipolar atom model after electron density database transfer. The mean energy values of different contact types between multipolar pseudoatoms were compared statistically to the contact enrichment ratios.

The analyses suggest that hydrogen bonds are often the most enriched and attractive interactions and are therefore a driving force in the crystal packing formation for organic molecules. The methodology also enables to compare different types of hydrogen bonds which are in competition within a crystal packing. The behavior of weaker interactions such as halogen bonds is less contrasted. The methodology is a way to rank the occurrence of given synthons and the impact in crystal engineering will be discussed.

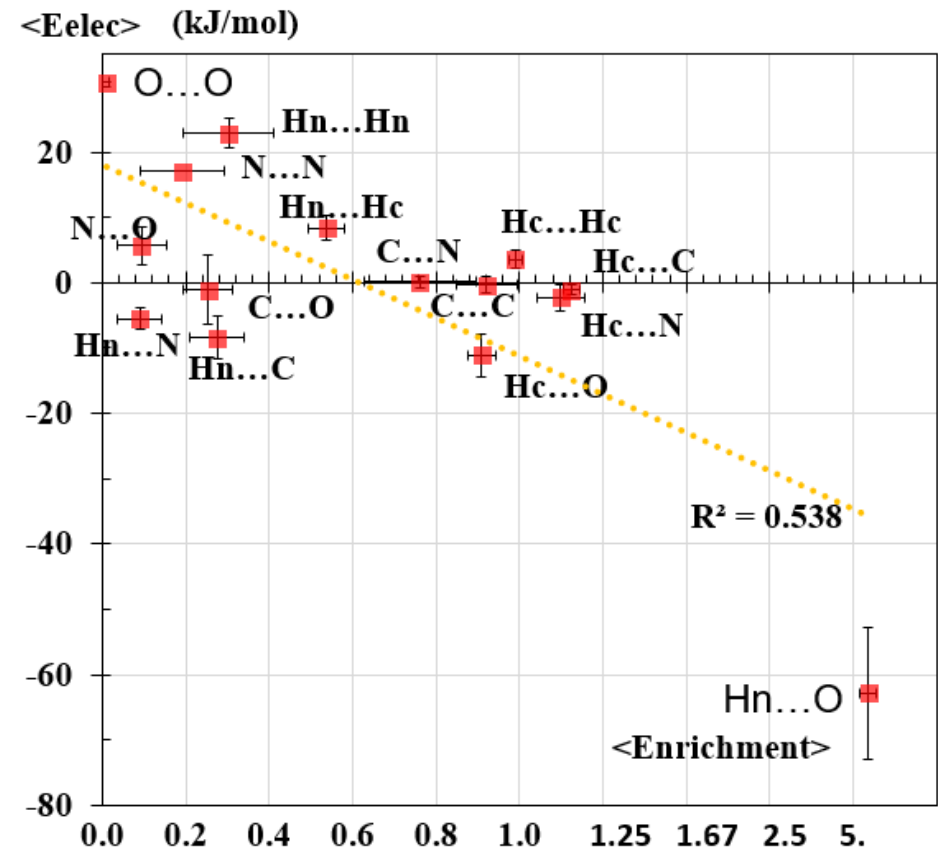

Figure 1.

Scatterplot of average electrostatic energy vs average contact enrichment ratio for 79 amide hydrocarbon molecules. Horizontal error bars correspond to the sample standard deviation of enrichment divided by 5 .

[1] Jelsch, C. \& Bibila Mayaya Bisseyou Y., IUCrJ 2017, 4, 158.

[2] Jelsch, C., Soudani, S. \& Ben Nasr C. IUCr J. 2015, 2, 327.

[3] Jelsch, C., Ejsmont, K. \& Huder, L. IUCr J. 2014, 1, 119.

[1] Margilies, L., Kramer, M. J., McCallum, R. W., Kycia, S., Haeffner, D. R., Lang, J. C. \& Goldman, A. I. (1999). Rev. Sci. Instrum. 70, 3554.

Keywords: atomic interactions; crystal packing ; multipolar atom model ; halogen bond 